\title{
CURVATURE, TRIAMETER, AND BEYOND
}

\author{
KARSTEN GROVE AND STEEN MARKVORSEN
}

\begin{abstract}
In its most general form, the recognition problem in Riemannian geometry asks for the identification of an unknown Riemannian manifold via measurements of metric invariants on the manifold. We introduce a new infinite sequence of invariants, the first term of which is the usual diameter, and illustrate the role of these global shape invariants in a number of recognition problems.
\end{abstract}

It is apparent that information about basic geometric invariants such as curvature, diameter, and volume alone does not suffice in order to characterize Riemannian manifolds in general. For this reason it is not only natural but necessary to pursue and investigate other metric invariants. Recently such investigations have included Gromov's filling radius (cf., e.g., [G, K1, K2, W]), excess invariants (cf., e.g., [GP2, O, PZ]), and Urysohn's intermediate diameters (cf., e.g., [U, G, K3]).

The purpose here is to introduce new metric invariants and announce related recognition theorems. Our main concern is an infinite sequence: diameter, triameter, quadrameter, quintameter, ..., etc., whose $n$th term is based on measurements on $(n+1)$-tuples of points. Precisely, if $(X$, dist $)$ is any compact metric space, the $q$-extent, $\mathrm{xt}_{q} X$, of $X$ is the maximal average distance between $q$ points in $X$, i.e.,

$$
\mathrm{xt}_{q} X=\max _{\left(x_{1}, \ldots, x_{q}\right)} \sum_{i<j} \operatorname{dist}\left(x_{i}, x_{j}\right) /\left(\begin{array}{l}
q \\
2
\end{array}\right), \quad x_{1}, \ldots, x_{q} \in X .
$$

With this definition, $\mathrm{xt}_{2} X=\operatorname{diam} X, \mathrm{xt}_{3} X=\operatorname{triam} X$, etc., and obviously

$$
\mathrm{xt}_{2} X \geq \mathrm{xt}_{3} X \geq \cdots \geq \mathrm{xt}_{q} X \geq \mathrm{xt}_{q+1} X \geq \cdots \geq \mathrm{xt} X,
$$

where $\mathrm{xt} X=\lim _{q} \mathrm{xt}_{q} X$ is called the extent of $X$. It is easy to see that

$$
\frac{1}{2} \operatorname{diam} X \leq \mathrm{xt} X<\operatorname{diam} X
$$

for any compact $X$ and that these inequalities are optimal. Somewhat surprisingly, however, it turns out that $x t X$ is related to the excess, exc $X$, as defined in [GP2]. Namely, if $X$ has almost minimal extent, its excess is almost zero. From this and several explicit computations (cf. [GM]), it appears that the above extent invariants are sensitive to asymmetries of a space and, therefore, should be thought of not as size invariants, but as global shape invariants in the same way that curvatures are thought of a local shape invariants.

Received by the editors November 5, 1991.

1980 Mathematics Subject Classification (1985 Revision). Primary 53C20, 51K10, 53C23.

Both authors were supported in part by the Danish Research Council; the first author was also supported by a grant from the National Science Foundation. 


\section{SPACES WITH LARGE EXTENTS}

The first step in the recognition program for a given metric invariant is to investigate its range when restricted to various subclasses of metric spaces (cf. $[\mathrm{G} 2, \mathrm{GM}])$. For the individual $q$-extents there are trivial optimal estimates like the one given for $\mathrm{xt} X$ above, even when restricted to Riemannian manifolds. This becomes an attractive problem, however, when a lower curvature bound is present, and thus local and global shape invariants are balanced against one another.

Specifically, for each $k \in \mathbb{R}$ and $R>0$, let $D_{k}^{n}(R)$ denote the closed metric $R$-ball in the simply connected $n$-dimensional complete space form $S_{k}^{n}$ of constant curvature $k$. For any closed Riemannian $n$-manifold $M$, whose sectional curvature and radius satisfy $\sec M \geq k$ and $\operatorname{rad} M \leq R$, standard Toponogov distance comparison yields

$$
\mathrm{xt}_{q} M \leq \mathrm{xt}_{q} D_{k}^{n}(R),
$$

for any integer $q \geq 2$. Recall that $\operatorname{rad} X \leq R$ if and only if $X=D(x, R)$ for some $x \in X$ (cf. [SY, GP4]).

When $k>0$ and $R \geq \pi / 2 \sqrt{k}$, it turns out that $\mathrm{xt}_{q} D_{k}^{n}(R)=\mathrm{xt}_{q} S_{k}^{n}=$ $\mathrm{xt}_{q}[0, \pi / \sqrt{k}]$ for all $q$, and all $q$-extenders, i.e., $q$-tuples of points realizing $\mathrm{xt}_{q}$, can be explicitly described (cf. [GM, N]). Moreover the inequalities (*) are optimal in this case. The following range/recognition theorem generalizes Toponogov's maximal diameter theorem (cf. [CE]):

Theorem A. Let $M$ be a closed Riemannian $n$-manifold, $n \geq 2$, with $\sec M \geq$ 1. Then $\mathrm{xt}_{q} M \leq \mathrm{xt}_{q} S_{1}^{n}$ for every $q \geq 2$.

If equality holds for some $q \geq 2$, then $M$ is isometric to $S_{1}^{n}$.

For any $\varepsilon>0$ and any $\pi / 2 \leq R \leq \pi$ there is a Riemannian manifold $M \simeq S^{n}$ with $\sec M \geq 1, \operatorname{rad} M \leq R$, and $\mathrm{xt}_{q} M \geq \mathrm{xt}_{q} S_{1}^{n}-\varepsilon$.

Note that since $\operatorname{diam} M \geq \mathrm{xt}_{q} M$ for any $q \geq 2$, the diameter sphere theorem [GS] implies that $M$ is homeomorphic to $S^{n}$ if $\mathrm{xt}_{q} M>\pi / 2$ in the above theorem.

In the remaining cases we only know $\mathrm{xt}_{q} D_{k}^{n}(R)$ when $q \leq n+1$ (cf. [GM, $\mathrm{T}, \mathrm{H}]$ ). Here $\mathrm{xt}_{n+1} D_{k}^{n}(R)$ is of particular interest because for $R<\pi / 4 \sqrt{k}$ (if $k>0)$ there is only one $(n+1)$-extender, namely, the vertices of the unique maximal, regularly inscribed $n$-simplex, $\Delta_{k}^{n}(R)$ in $D_{k}^{n}(R)$. Here without the loss of generality we may assume $R=1$. If $r(n, k)$ is the radius of the largest ball inscribed in $\Delta_{k}^{n}=\Delta_{k}^{n}(1)$, then $r(n, \cdot):\left(-\infty,(\pi / 2)^{2}\right) \rightarrow(0,1)$ is a strictly increasing continuous function for each $n \geq 2$. Let $k(n)$ be determined by $r(n, k(n))=1 / 2$. The optimality question of $(*)$ is then resolved for $q=n+1$ according to the following.

Theorem B. Fix an integer $n \geq 2$ and $k<\left(\frac{\pi}{4}\right)^{2}$. For any closed Riemannian $n$-manifold $M$ with $\sec M \geq k$ and $\operatorname{rad} M \leq 1$,

$$
\mathrm{xt}_{n+1} M<\mathrm{xt}_{n+1} D_{k}^{n}(1),
$$

and this inequality is optimal if and only if $k \leq k(n)$.

There is an $\varepsilon(n)>0$, so that if $k=k(n)$ and 


$$
\mathrm{xt}_{n+1} M \geq \mathrm{xt}_{n+1} D_{k(n)}^{n}(1)-\varepsilon(n),
$$

then $M$ is homeomorphic to $S^{n}$.

In contrast to the proof of Theorem A, this result is proved using convergence techniques. It follows from these techniques via [GPW] (cf. also a recent result of Perelman announced in [BGP]) that there are at most finitely many topological types of manifolds $M$ as in Theorem B, for which $\mathrm{xt}_{n+1} M \geq$ $\mathrm{xt}_{n+1} D_{k}^{n-1}(1)+\varepsilon$, for any fixed $\varepsilon>0$.

Once it has been observed that the double $D \Delta_{k}^{n}=\Delta_{k}^{n} \amalg \Delta_{k}^{n} /\left(\partial \Delta_{k}^{n} \sim \partial \Delta_{k}^{n}\right)$ of the simplex $\Delta_{k}^{n}$ has curvature curv $D \Delta_{k}^{n} \geq k$ in distance comparison sense and $\operatorname{rad} D \Delta_{k}^{n}=1$ when $k \leq k(n)$, the optimality statement in Theorem B is fairly trivial. The hard part is to prove the recognition statement when $k=k(n)$ and the nonoptimality statement when $k>k(n)$. This, on the other hand, follows from Theorem $\mathrm{C}$ below together with results and tools developed in [GP3, GPW].

Following the terminology of [BGP], a FSCBB, or an Aleksandrov space is a complete inner metric space with finite Hausdorff dimension, which is curved from below in (local) distance comparison sense.

Theorem C. Let $X$ be an n-dimensional Aleksandrov space with curv $X \geq k$, $k<\left(\frac{\pi}{4}\right)^{2}, \operatorname{rad} X=1$, and $\mathrm{xt}_{n+1} X=\mathrm{xt}_{n+1} D_{k}^{n}(1)$. Then

(i) There is an isometric embedding of $\Delta_{k}^{n}$ in $X$ with totally geodesic interior;

(ii) If $X$ is a Poincaré duality space then $k \leq k(n)$;

(iii) If $X$ is a Poincare duality space and $k=k(n)$, then $X$ is isometric to $D \Delta_{k(n)}^{n}$.

The essential new technical tool used in the proof of Theorem $C$ is the following analogue of the rigidity version of Toponogov's distance comparison theorem for Aleksandrov spaces.

Theorem D. Let $X$ be an Aleksandrov space with curv $X \geq k$. For any pair $\left(p_{0}, c_{0}\right)$, where $c_{o}$ is a minimal geodesic in $X$ with end points $p_{1}, p_{2}$ and $p_{0} \notin c_{0}$, let $\left(\bar{p}_{0}, \bar{c}_{0}\right)$ be the corresponding pair in $S_{k}^{2}$, i.e. $\operatorname{dist}\left(p_{i}, p_{j}\right)=$ $\operatorname{dist}\left(\bar{p}_{i}, \bar{p}_{j}\right), 0 \leq i<j \leq 2$ (if $k>0$ assume all distances $<\pi / \sqrt{k}$ ). Then for corresponding interior points $q \in c_{0}, \bar{q} \in \bar{c}_{0}$, we have

(i) $\operatorname{dist}\left(p_{0}, q\right) \geq d\left(\bar{p}_{0}, \bar{q}\right)$, and

(ii) if equality holds, any minimal geodesic $c_{q}$ from $p_{0}$ to $q$ spans together with $c_{0}$ a unique triangular surface isometric to the one spanned by $\left(\bar{p}_{0}, \bar{c}_{0}\right)$ in $S_{k}^{2}$, and whose interior is totally geodesic.

Part (i) of this global distance comparison theorem was proved in [BGP]. A rigidity statement for hinges as in Riemannian geometry (cf. [CE, G1]) follows by applying (ii) above twice.

In the generality of Theorem $\mathrm{C}$ as stated above, we also need a new metric characterization of $S_{1}^{n}$. In order to describe this, let the q-packing radius, pack $_{q} X$, of $X$ be the largest $r$ so that there are $q$ disjoint open $r$-balls in $X$, i.e.,

$$
2 \operatorname{pack}_{q} X=\max _{\left(x_{1}, \ldots, x_{q}\right)} \min _{i<j} \operatorname{dist}\left(x_{i}, x_{j}\right), \quad x_{i}, \ldots, x_{q} \in X .
$$


Theorem E. Let $X$ be an $n$-dimensional Aleksandrov space with $\operatorname{curv}(X) \geq 1$. Then

$$
\operatorname{pack}_{n+2}(X) \leq \operatorname{pack}_{n+2}\left(S_{1}^{n}\right)
$$

and equality holds if and only if $X$ is isometric to $S_{1}^{n}$.

This characterization together with Yamaguchi's fibration theorem [Y] also yields the following pinching theorem of independent interest.

Corollary F. For each integer $n \geq 2$ there is an $\varepsilon=\varepsilon(n)>0$ such that any Riemannian n-manifold $M$ with $\sec M \geq 1$ and $\operatorname{pack}_{n+2}(M) \geq \operatorname{pack}_{n+2}\left(S_{1}^{n}\right)-\varepsilon$ is diffeomorphic to $S^{n}$.

We conclude with another pinching-or rather recognition theorem. As in our previous results above, this is also based on a complete metric classification of a certain class of Aleksandrov spaces. In this case the class consists of all $n$-dimensional Aleksandrov spaces $X$ with $\operatorname{curv}(X) \geq 1$ and $\operatorname{diam}(X)=$ $\mathrm{xt}_{n+1}(X)=\pi / 2$. Rather than giving the list here, we point out that for each $n$ only two of them are Poincáre duality spaces, namely, $\mathbb{R} P_{1}^{n}=S_{1}^{n} / \mathbb{Z}_{2}$ and the double spherical simplex $D \Delta_{1}^{n}$. From this one derives

Theorem G. For each $n \geq 2$ there is an $\varepsilon=\varepsilon(n)>0$ with the following property. Any closed Riemannian $n$-manifold $M$ with $\sec M \geq 1$ and $\mathrm{xt}_{n+1}(M) \geq \pi / 2-\varepsilon$ is either diffeomorphic to $\mathbb{R} P^{n}$ or homeomorphic to $S^{n}$. Moreover, there are metrics on $M \sim S^{n}, \mathbb{R} P^{n}$ with $\sec M \geq 1$, diam $M \leq \pi / 2$, and $\mathrm{xt}_{n+1}(M)$ arbitrarily close to $\pi / 2$.

This result can be viewed as a generalization of the main results in [GP1, OSY].

Details and further applications will be published in [GM].

It is our pleasure to thank S. Ferry for helpful suggestions related to the proof of Theorem C.

\section{REFERENCES}

[BGP] Y. Burago, M. Gromov, and G. Perelman, Aleksandrov's spaces with curvatures bounded from below I, Uspekhi Mat. Nauk (to appear).

[CE] J. Cheeger and D. G. Ebin, Comparison theorems in Riemannian geometry, North-Holland Math Library, vol. 9, North-Holland, Amsterdam, 1975.

[GG] D. Gromoll and K. Grove, A generalization of Berger's rigidity theorem for positively curved manifolds, Ann. Sci. École. Norm. Sup. 20 (1987), 227-239.

[G] M. Gromov, Filling Riemannian Manifolds, J. Differential Geom. 18 (1983), 1-148.

[G1] K. Grove, Metric differential geometry, Differential Geometry (V. L. Hansen, ed.), Lecture Notes in Math, vol. vol. 1263, Springer-Verlag, 1987, pp. 171-227.

[G2] - Metric and topological measurements of manifolds, Proc. International Congress of Mathematicians, Kyoto, Japan 1990, Springer-Verlag, 1991, pp. 511-519.

[GM] K. Grove and S. Markvorsen, Metric invariants for the Riemannian recognition program via Aleksandrov geometry, preprint.

[GP1] K. Grove and P. Petersen V, Homotopy types of positively curved manifolds with large volume, Amer. J. Math. 110 (1988), 1183-1188.

[GP2] _ A pinching theorem for homotopy spheres, J. Amer. Math. Soc. 3 (1990), 671-677.

[GP3] _ Manifolds near the boundary of existence, J. Differential Geom. 33 (1991), 379394.

[GP4] , Volume comparison à la Aleksandrov, Acta. Math. (to appear).

[GPW] K. Grove, P. Petersen, and J.-Y. Wu, Geometric finiteness theorems via controlled topology, Invent. Math. 99 (1990), 205-213. 
[GS] K. Grove and K. Shiohama, A generalized sphere theorem, Ann. of Math. (2) 106 (1977), 201-211.

[H] E. Hille, Remarks on transfinite diameters, J. Analyse Math. 14 (1965), 209-224.

[K1] M. Katz, The filling radius of two-point homogeneous spaces, J. Differential Geom. 18 (1983), 505-511.

[K2] , The rational filling radius of complex projective spaces, preprint.

[K3] The first diameters of 3-manifolds of positive scalar curvature, Proc. Amer. Math. Soc. 104 (1988), 591-595.

[N] F. Nielsen, On the sum of distances between $n$ points on the sphere (Danish), Nordisk Tidskr. Mat. 13 (1965), 45-50.

[O] Y. Otsu, On manifolds of small excess, Amer. J. Math. (to appear).

[OSY] Y. Otsu, K. Shiohama, and T. Yamaguchi, $A$ new version of differentiable sphere theorem, Invent. Math. 98 (1989), 219-228.

[PZ] P. Petersen and Zhu, An excess sphere theorem, Ann. Sci. Éc. Norm. Sup. (to appear).

[R] W. Rinow, Die Innere Geometrie der Metrischen Räume (1961), Springer-Verlag, 1961.

[SY] K. Shiohama and T. Yamaguchi, Positively curved manifolds with restricted diameters, Geometry of Manifolds (K. Shiohama, ed.), Perspect. in Math, vol. 8, Academic Press, Boston, MA, 1989, pp. 345-350.

[T] F. Toth, On the sum of distances determined by a pointset, Acta. Math. Acad. Sci. Hungar. 7 (1957), 397-401.

[U] P. Urysohn, Notes supplémentaires, Fund. Math. 8 (1926), 352-356.

[W] F. Wilhelm, On the filling radius of positively curved manifolds, Invent. Math. 107 (1992), 653-668.

[Y] T. Yamaguchi, Collapsing and pinching under a lower curvature bound, Ann. of Math. (2) 133 (1991), 317-357.

Department of Mathematics, University of Maryland, College Park, Maryland 20742-0001

E-mail address: kng@math.umd.edu

Mathematics Institute, Technical University of Denmark, 2800 Lyngby, Denmark

E-mail address: steen@mat.dth.dk 